\title{
A RELAÇÃO ENTRE A URBANIZAÇÃO E A EPIDEMIA DA OBESIDADE A NÍVEL MUNDIAL E EM PORTUGAL
}

\section{The relation between urbanization and obesity epidemic worldwide and in Portugal}

\author{
Margarida Pereira \\ mmiguel06@gmail.com \\ Centro de Investigação em Antropologia e Saúde, Universidade de Coimbra, Calçada Martim de \\ Freitas, Edifício de São Bento, 3000-456 Coimbra \\ Helena Nogueira \\ helenamarquesnogueira@gmail.com \\ Centro de Investigação em Antropologia e Saúde, Universidade de Coimbra, Calçada Martim de \\ Freitas, Edifício de São Bento, 3000-456 Coimbra \\ Paulo Nossa \\ paulonnossa@gmail.com \\ Centro de Estudos de Geografia e Ordenamento do Território - 3004-530, Coimbra, Portugal
}

\begin{abstract}
RESUMO: Enquadramento: A obesidade é um grave problema de saúde pública em todo o mundo devido ao seu impacto negativo na saúde dos indivíduos e aos altos custos para os sistemas de saúde. O objetivo deste estudo foi avaliar a relação entre a proporção de população urbana e a prevalência de obesidade em todo o mundo e em Portugal. Métodos: Trata-se de um estudo transversal que utilizou dados disponíveis gratuitamente quanto à percentagem de população urbana e prevalência de obesidade, em nível global e nacional. Os dados recolhidos foram organizados por país, a nível mundial e, por regiões em Portugal. Posteriormente, os dados foram mapeados e os resultados foram representados através de gráficos de dispersão, com linha de regressão e o respetivo coeficiente de determinação. Resultados: Os resultados deste estudo mostram que existe uma correlação entre obesidade e urbanização $(r=0,502, p<0,01)$ em todo o mundo e em Portugal. Conclusões: As evidências sugerem que a obesidade poderá ser determinada por características ambientais, como o nível de urbanização do local onde as pessoas vivem e trabalham. A população urbana tende a ser menos ativa e ingerir mais alimentos altamente calóricos, o que leva ao sobrepeso e à obesidade
\end{abstract}

Palavras-chave: Urbanização; Obesidade; Epidemia; Mundial; Portugal

ABSTRACT: Objectives: This study main goal is to assess the relation between the urban population and obesity prevalence worldwide and in Portugal thus, contributing to a broader understanding of the link between both constructs. Study Design: This is a cross-sectional study once it analyses data collected from a population, at a specific point in time. Methods: This study uses freely available data regarding the percentage of urban population and obesity prevalence, at the global and national level. All information collected was organized and joined by country and by Portugal's region level. Afterwards, choropleth maps were created, and Scatter Plot with Regression Line and the respective Determinant Coefficient were computed. Results: This study results show that there is a correlation between obesity and urbanization $(r=0.502, p<0.01)$ worldwide and in Portugal. Conclusions: Evidence suggests that obesity might also be determined by environmental features such as the urbanization level of the place where people live and work. Urban population tend to be less physically active and ingest more highcaloric food which leads to overweight and obesity.

Key Words: Urbanization; Obesity; Epidemic; Worldwide; Portugal

REVISTA GEONORTE, V.9, N.32, p.06-23, 2018.

DOI: 10.21170/geonorte.2018.V.9.N.32.06.23

(ISSN 2237 - 1419) 


\section{INTRODUÇÃO}

A obesidade tem aumentado a nível mundial nas últimas quatro décadas. Em 2013 a prevalência de excesso de peso e/ou obesidade mundial (índice de massa corporal (IMC) acima de $25 \mathrm{~kg} / \mathrm{m}^{2}$ ), em adultos com mais de 18 anos, era superior a $36,9 \% \mathrm{em}$ homens, 38,0\% em mulheres. Em rapazes e raparigas com menos de 18 anos, a prevalência de obesidade a nível mundial era $23,8 \%$ e $22,6 \%$, respetivamente [1]. Em Portugal, $28,7 \%$ dos rapazes e $27,1 \%$ das raparigas com menos de 20 anos de idade tinham excesso de peso ou obesidade assim como, $63,8 \%$ dos homens e $54,6 \%$ das mulheres com 20 ou mais anos [1]. Este cenário é bastante preocupante porque, como é sabido, a obesidade é um fator de risco major para várias doenças crónicas incluindo a diabetes, doenças cardiovasculares, cancro e pode levar a morte prematura [2, 3]. A obesidade diminui a qualidade de vida dos indivíduos e é responsável por uma fatia considerável do orçamento dos cuidados de saúde $[4,5]$. Por conseguinte, um pouco por todo o mundo a prevenção da obesidade tem-se tornado uma prioridade nas agendas de políticas de saúde pública.

Inicialmente, a obesidade foi considerada como uma consequência direta dos comportamentos individuais. Contudo, esta abordagem tem mudado ao longo do tempo [2] principalmente devido ao aumento continuo da prevalência da obesidade apesar das tentativas no sentido de reverter esta tendência [1] através de intervenções direcionadas ao indivíduo [6]. De facto, a evidência recente mostra que a obesidade é determinada por um sistema complexo de fatores que interagem de forma dinâmica entre si $[6,7]$.

Um dos determinantes da obesidade é o processo de urbanização $[5,6]$ que é produto da industrialização e do desenvolvimento económico [3]. Como esperado há algum tempo, na atualidade a maioria da população mundial vive em cidades ou em áreas densamente urbanizadas, independentemente do nível de desenvolvimento do país [3]. A urbanização impacta a saúde de diversas formas nomeadamente através da mudança do estilo de vida geral, isto é, nas sociedades modernas as pessoas tendem a ser cada vez mais sedentárias devido ao tipo de trabalho e de transporte que utilizam, e ingerem comida cada vez mais processada e altamente calórica uma vez que comem maioritariamente foram de casa [3]. Assim, a evolução da sociedade no sentido de diminuir a necessidade de ser ativo fisicamente, juntamente com a evolução da industria alimentar têm contribuído para o aumento do excesso de peso e obesidade [5, 8].

Em resumo, a urbanização e a obesidade estão ambas a aumentar a nível mundial e a designada "epidemia da obesidade" não é só um problema dos países de elevada renda económica, como se acreditava. Ao invés, a prevalência da obesidade está também a aumentar nos países de média e baixa renda económica [9, 10], em particular nas áreas urbanas [5, 8].

O rápido processo de urbanização acarreta vários problemas como a superlotação, desemprego, construções de fraca qualidade ou problemas de trafego [3]. Nestas áreas, o planeamento urbano torna-se uma tarefa complexa que responde 
essencialmente a questões imediatas e cujas intervenções tendem a negligenciar preocupações com as questões de saúde. Sui (2013, p.79) afirma:

\begin{abstract}
"It is almost a cliché nowadays to say that we shape our cities and our cities will shape us. But the truth of such a cliché is clearly manifested by the relationship between obesity and urban sprawl. The city and its people in general, urban forms and the well-being of urban residents in particular are closely linked. So long as we continue to build more parking lots instead of parks, neither obesity nor urban sprawl can be mitigated significantly."
\end{abstract}

A relação entre a obesidade e a urbanização é subtil, portanto é necessária mais evidência por forma a sustentar estratégias informadas no combate à obesidade. Desta forma, este trabalho tem como principal objetivo providenciar um enquadramento geral, a nível global e nacional, da relação entre urbanização e obesidade. Mais especificamente, este trabalho pretende contribuir para um conhecimento alargado entre a relação referida anteriormente através da quantificação da associação entre a prevalência da obesidade e a percentagem de pessoas a residir em áreas urbanas a nível mundial e em Portugal.

\title{
Obesidade e Urbanização
}

Na década de 1980 a abordagem ao conceito de corpo ideal, comummente utilizada pelos médicos para avaliar a composição corporal, foi substituída pelo rácio entre o peso e a altura, isto é, o Índice de Massa Corporal (IMC) [2]. Os pontos de corte do IMC para o excesso de peso e a obesidade são 25-30 e acima dos 30, respetivamente. Mais tarde, em 1997, a Organização Mundial da Saúde (OMS) reconheceu formalmente que a proporção de população com excesso de peso e obesidade era uma epidemia [2].

Atualmente mais de $50 \%$ da população total a nível mundial, mais especificamente $54,293 \%$ [11], vive em áreas urbanas onde é difícil praticar atividade física devido ao tráfego, à baixa qualidade do ar, à falta de segurança e à sobrelotação [12]. Patil [3] define urbanização como "(...) a process that leads to the growth of cities due to industrialization and economic development and that leads to urban-specific changes in specialization, labour division, and human behaviours." Viver em áreas urbanas não só aumenta os comportamentos sedentários, como também aumenta o acesso a comida altamente calórica, o que contribui para a epidemia da obesidade [3].

Em 2002, o grupo de trabalho International Obesity Force Task (IOTF), desenvolveu um enquadramento conceptual no qual é claro que a urbanização tem um papel importante no aumento do excesso de peso e obesidade a nível mundial, ainda que indiretamente. O IOTF considera que a urbanização, assim com o crescimento económico, a modernização e globalização especificamente da comida estão a "(...) societal and environmental forces thought to underlie the [obesity] epidemic." [13].

Barry M. Popkin foi o primeiro investigador a utilizar o conceito de Transição Nutricional e a apresenta-lo em paralelo com as transições demográfica e 


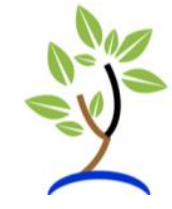

epidemiológica [14]. As transições demográfica, epidemiológica e nutricional partilham as formas pelas quais as populações transitam dum padrão para o outro. Estas transições focam-se na melhoria do planeamento familiar, no controlo de doenças infeciosas e na prevenção da fome por forma a reduzir a mortalidade e aumentar a esperança de vida.

Popkin and Gordon-Larsen [15], p. S3 descreve o mecanismo destas transições entre as fases 3, 4 e 5 da transição nutricional da seguinte forma:

\begin{abstract}
"In Stage 3, famine begins to recede as income rises. In Stage 4, changes in diet and activity pattern lead to the emergence of new disease problems and increased disability. In Stage 5, behavioural change begins to reverse the negative tendencies and make possible a process of 'successful aging'. The changes are all driven by a range of factors, including urbanization, economic growth, technical change, and culture. For convenience, the patterns can be thought of as historical developments; however, 'earlier' patterns are not restricted to the periods in which they first arose, but continue to characterize certain geographic and socioeconomic (SES) subpopulations."
\end{abstract}

Assim, neste mecanismo, a urbanização é considerada tão importante como o processamento dos alimentos, as alterações das atividades no tempo de lazer e nos padrões alimentares ou até na composição corporal $[15,16]$.

Em síntese, as áreas urbanas são maioritariamente constituídas por áreas com um uso de solo densamente edificado e normalmente sobrelotado o que pode diminuir o sentimento de segurança [17]. Nestas áreas, o principal meio de transporte é passivo, ou seja, normalmente os indivíduos deslocam-se de carro, táxi ou transportes públicos logo, a intensidade do tráfego é consideravelmente mais elevada do que noutras áreas. O estilo de vida urbano é predominantemente sedentário uma vez que os empregos nas áreas urbanas são realizados sentados (utilizando o computador por exemplo) o que por sua vez diminui a atividade física [18]. Além disso, o consumo de alimentos altamente calóricos predomina em áreas urbanas [18]. Todos estes aspetos combinados contribuem decisivamente para os indivíduos terem excesso de peso e obesidade.

Até ao momento, este é o único estudo que se foca na associação entre urbanização e obesidade através da quantificação da relação entre os dois construtos utilizando dados disponíveis gratuitamente a nível global e nacional. No geral, os estudos sobre obesidade e urbanização são realizados ao nível da cidade, quer em Portugal [17, 19] quer noutros países $[9,20]$.

\title{
Materiais e Métodos
}

Dados: fontes e metainformação

$\mathrm{Na}$ Tabela 1 as variáveis utilizadas neste trabalho estão listadas assim como a sua definição, ano e fonte. 
Tabela 1 - Fontes dos Dados e Metainformação

\begin{tabular}{|c|c|c|c|c|c|}
\hline Nível & Variável & Indicador & Definição & Ano & Fonte \\
\hline & $\begin{array}{l}\text { \% } \\
\text { População } \\
\text { Residente } \\
\text { em Áreas } \\
\text { Urbanas }\end{array}$ & $\begin{array}{l}\text { População } \\
\text { Urbana (\% do } \\
\text { total) }\end{array}$ & $\begin{array}{l}\text { A população urbana refere-se a } \\
\text { pessoas que vivem em áreas urbanas, } \\
\text { conforme definido pelos institutos de } \\
\text { estatística nacionais. Os dados são } \\
\text { recolhidos e afinados pela Divisão de } \\
\text { População das Nações Unidas. }\end{array}$ & 2014 & Banco Mundial \\
\hline Mundo & $\begin{array}{l}\text { \% de } \\
\text { População } \\
\text { com um } \\
\text { IMC }>30\end{array}$ & $\begin{array}{l}\text { Obesidade } \\
\text { (Índice de } \\
\text { Massa } \\
\text { Corporal >= } \\
30 \text { ), } \\
\text { estandardizado } \\
\text { pela idade } \\
\text { estimada por } \\
\text { país }\end{array}$ & $\begin{array}{l}\text { Estimativas para IMC, sobrepeso e } \\
\text { obesidade, para o ano-padrão } 2010 \text {, } \\
\text { serviram como base para relatórios } \\
\text { sobre os objetivos voluntários globais de } \\
\text { DNTs, em 2014. As estimativas } \\
\text { ajustadas baseiam-se em dados } \\
\text { agregados fornecidos à OMS e ao } \\
\text { Grupo de Trabalho sobre o Peso Global } \\
\text { dos Fatores de Risco Metabólico das } \\
\text { Doenças Crónicas, obtidos por meio de } \\
\text { revisão de literatura publicada e não } \\
\text { publicada. Os critérios de inclusão para } \\
\text { a análise de estimativa incluíram dados } \\
\text { provenientes de uma amostra aleatória } \\
\text { da população geral, com métodos de } \\
\text { pesquisa claramente indicados } \\
\text { (incluindo tamanhos de amostra) e } \\
\text { definições de fatores de risco. Os } \\
\text { ajustes foram feitos para os seguintes } \\
\text { fatores para que o mesmo indicador } \\
\text { pudesse ser reportado para um ano } \\
\text { padrão (neste caso, } 2010 \text { e } 2014 \text { ) em } \\
\text { todos os países: definição padrão do } \\
\text { fator de risco, conjunto padrão de } \\
\text { grupos de idade para indicador; ano de } \\
\text { referência padrão e representatividade } \\
\text { da população. Utilizando técnicas de } \\
\text { modelação de regressão, foram } \\
\text { produzidas taxas ajustadas para cada } \\
\text { indicador. Para permitir a comparação } \\
\text { entre os países, foram produzidas } \\
\text { estimativas comparáveis padronizadas } \\
\text { por idade. Isso foi feito ajustando as } \\
\text { estimativas brutas à População Padrão } \\
\text { da OMS (4), que reflete a estrutura } \\
\text { etária e por sexo da maioria dos países } \\
\text { de renda baixa e média. Isto corrige as } \\
\text { diferenças na estrutura etária / de sexo } \\
\text { entre os países. A incerteza nas } \\
\text { estimativas foi analisada tendo em } \\
\text { conta o erro de amostragem e a } \\
\text { incerteza devido à modelação } \\
\text { estatística. As estimativas incluídas nos } \\
\text { grupos Regionais da OMS e }\end{array}$ & 2014 & $\begin{array}{l}\text { Organização } \\
\text { Mundial de Saúde }\end{array}$ \\
\hline
\end{tabular}




\begin{tabular}{|c|c|c|c|c|c|}
\hline & & & $\begin{array}{l}\text { Rendimentos do Banco Mundial são as } \\
\text { estimativas comparáveis padronizadas } \\
\text { por idade. Os dados reportados a partir } \\
\text { de outubro de } 2014 \text { foram incluídos no } \\
\text { processo de estimativa. (Fonte: } \\
\text { http://www.who.int/gho/ncd/methods/en/, } \\
\text { 07/12/2016) }\end{array}$ & & \\
\hline & $\begin{array}{l}\text { Classe } \\
\text { Económica } \\
\text { por País }\end{array}$ & $\begin{array}{l}\text { Classe } \\
\text { Económica por } \\
\text { País por } \\
\text { Renda }\end{array}$ & $\begin{array}{l}\text { Para o ano fiscal de } 2017 \text {, as } \\
\text { economias de baixa renda são definidas } \\
\text { como aquelas com um PIB per capita, } \\
\text { calculado pelo método do Atlas do } \\
\text { Banco Mundial, de US } \$ 1.025 \text { ou } \\
\text { menos em } 2015 ; \text { economias de renda } \\
\text { média baixa são aquelas com um PIB } \\
\text { per capita entre US } \$ 1.026 \text { e US } \$ \\
4.035 ; \text { as economias de renda média } \\
\text { alta são aquelas com um PIB per capita } \\
\text { entre US } \$ 4.036 \text { e US } \$ 12.475 ; \\
\text { economias de alta renda são aquelas } \\
\text { com um PIB per capita de US } \$ 12.476 \\
\text { ou mais. }\end{array}$ & 2017 & Banco Mundial \\
\hline \multirow[t]{2}{*}{ Portugal } & $\begin{array}{l}\text { \% de } \\
\text { População } \\
\text { com um } \\
\text { IMC > } 30\end{array}$ & $\begin{array}{l}\text { População } \\
\text { Residente com } \\
18 \text { ou mais } \\
\text { anos por } \\
\text { classe de IMC, } \\
\text { sexo e grupo } \\
\text { de idade }\end{array}$ & $\begin{array}{l}\text { Índice de massa corporal: índice } \\
\text { internacional adotado pela OMS para } \\
\text { determinar se um indivíduo está abaixo } \\
\text { do peso, com peso normal, com } \\
\text { sobrepeso ou obesidade. O índice de } \\
\text { massa corporal é o quociente do peso } \\
\text { de uma pessoa em quilogramas e o } \\
\text { quadrado da sua altura em metros. } \\
\text { Classificação do índice de massa } \\
\text { corporal: baixo peso (IMC }<18,5 \mathrm{~kg} / \\
\left.\mathrm{m}^{2}\right) ; \text { Peso normal (IMC } \geq 18,5 \mathrm{~kg} / \mathrm{m}^{2} \text { e } \\
\left.<25 \mathrm{~kg} / \mathrm{m}^{2}\right) ; \text { Excesso de peso grau I } \\
\left(\mathrm{IMC} \geq 25 \mathrm{~kg} / \mathrm{m}^{2} \text { e }<27 \mathrm{~kg} / \mathrm{m}^{2}\right) ; \\
\text { Excesso de peso grau II (IMC } \geq 27 \mathrm{~kg} / \\
\left.\mathrm{m}^{2} \mathrm{e}<30 \mathrm{~kg} / \mathrm{m}^{2}\right) ; \mathrm{E} \text { obesidade }(\mathrm{IMC} \geq \\
\left.30 \mathrm{~kg} / \mathrm{m}^{2}\right) .\end{array}$ & $\begin{array}{l}2005 / 6 \\
\text { e } 2014\end{array}$ & $\begin{array}{l}\text { Inquérito Nacional } \\
\text { de Saúde (Instituto } \\
\text { Nacional de } \\
\text { Estatística) }\end{array}$ \\
\hline & $\begin{array}{l}\text { Densidade } \\
\text { de } \\
\text { População } \\
\text { Urbana }\end{array}$ & $\begin{array}{l}\text { Densidade } \\
\text { Populacional }\end{array}$ & $\begin{array}{l}\text { (No. / km²) por Local de residência } \\
\text { (NUTS - 2013) e Tipologia de áreas } \\
\text { urbanas; Anual }\end{array}$ & $\begin{array}{l}2011 \text { e } \\
2016\end{array}$ & $\begin{array}{l}\text { Instituto Nacional de } \\
\text { Estatística }\end{array}$ \\
\hline
\end{tabular}

Os dados no formato de shapefile, nomeadamente os limites administrativos dos países e as delimitações das regiões portuguesas foram retirados de http://thematicmapping.org/downloads/world borders.php http://www.dgterritorio.pt/cartografia e geodesia/cartografia/carta administrativa ofi cial de portugal caop/caop em vigor/, respetivamente.

Os dados mais recentes disponíveis gratuitamente sobre a percentagem de população urbana e a percentagem de indivíduos com um IMC superior a 30 foi recolhido a nível global e nacional. A nível global os dados recolhidos são de 2014, como se pode verificar na Tabela 1 exceto a informação sobre a classe económica dos países que 
foi recolhida no site do Banco Mundial e é relativa ao ano de 2017. Ao nível nacional a informação sobre o IMC foi recolhida no Inquérito Nacional da Saúde de 2005/6 e 2014 e a informação sobre a densidade da população urbana foi recolhida dos censos de 2011 e das projeções anuais do Instituto Nacional de Estatísticas de 2016.

Existem algumas limitações no que diz respeito à utilização desta informação. Por exemplo, a informação sobre o IMC da população é recolhida utilizando amostras populacionais na maioria dos países, no entanto, a tendência geral dos elevados níveis de obesidade é clara. Outra limitação relaciona-se com informação em falta para alguns países o que poderá ter algum impacto nos resultados.

\section{Análise da informação}

Toda a informação recolhida foi organizada em formato exce/ antes de ser importada para ambiente ArcGIS e SPSS. Depois, a informação sobre a população urbana e obesidade foi unida numa só base de dados utilizando a ferramenta adequada em ArcMap e, evitando assim os duplicados e garantindo a qualidade dos dados. Os mapas foram criados utilizando esta base de dados e, a classificação de cada variável calculou-se para o mapa mundial em quatro classes de acordo com as frequências e, em três classes para as regiões portuguesas. As análises estatísticas foram realizadas em SPSS e o significado estatístico considerado foi o $p=0.05$.

\section{Resultados}

\section{Mundial}

A percentagem média mundial de pessoas a viver em áreas urbanas é $56,64 \%$ e a percentagem média mundial de população com um IMC superior a 30 é 19,11\% (Tabela 2, análise de 187 países). No geral, países com alta renda económica apresentam percentagens altas de população urbana e de população com um IMC acima de 30.

Tabela 2 - Descrição Estatística Mundial por Classe Económica do País

\begin{tabular}{|l|l|l|l|}
\hline Classes Económicas & N & $\begin{array}{l}\text { \% População } \\
\text { residente em } \\
\text { Áreas Urbanas }\end{array}$ & $\begin{array}{l}\text { \% População } \\
\text { com um IMC > 30 }\end{array}$ \\
\hline Economias de Baixa Renda & a) & 32.73 & 5.39 \\
\hline Economias de Média a Baixa Renda & 50 & 43.53 & 14.64 \\
\hline Economias de Média a Alta Renda & 53 & 62.63 & 23.19 \\
\hline Economias de Alta Renda & b) & 74.63 & 26.87 \\
\hline Total & $\mathbf{1 8 7}$ & $\mathbf{5 6 . 6 4}$ & $\mathbf{1 9 . 1 1}$ \\
\hline
\end{tabular}




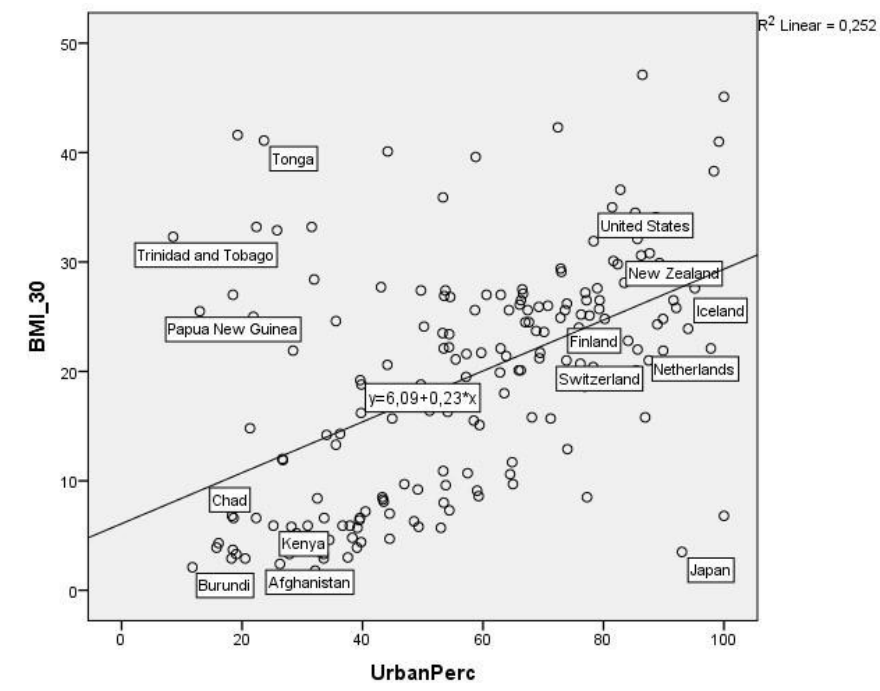

Fig. 1 - Scatter Plot com a Linha de Regressão e o respetivo Coeficiente de Determinação (\% População com IMC > 30 e \% População que vive em Áreas Urbanas) - Países do Mundo

Os resultados deste estudo mostram que há uma correlação entre obesidade e a urbanização $(r=0.502, p<0.01)$ o que significa que o aumento da percentagem de população que vive em áreas urbana é acompanhado pelo aumento da população com um IMC > 30, de forma significativa. Na verdade, o coeficiente de determinação representado no scatter plot informa que um pouco acima de $25 \%$ da variação da percentagem de população com um IMC acima de 30 poderá dever-se à variação da percentagem da população que vive em áreas urbanas (Fig. 1).

Os países de alta renda económica tais como os EUA, a Nova Zelândia ou a Islândia apresentam elevadas percentagens de população com um IMC acima de 30 assim como elevadas percentagens de população que vive em áreas urbanas, enquanto que países como o Burundi ou o Quénia apresenta os valores mais baixos de ambas as variáveis analisadas. No entanto, é também possível identificar vários países de baixa renda económica com baixas percentagens de população a viver em áreas urbanas, mas com elevadas percentagens de população com um IMC acima de 30, nomeadamente a Papua Nova Guiné ou o Tonga. 


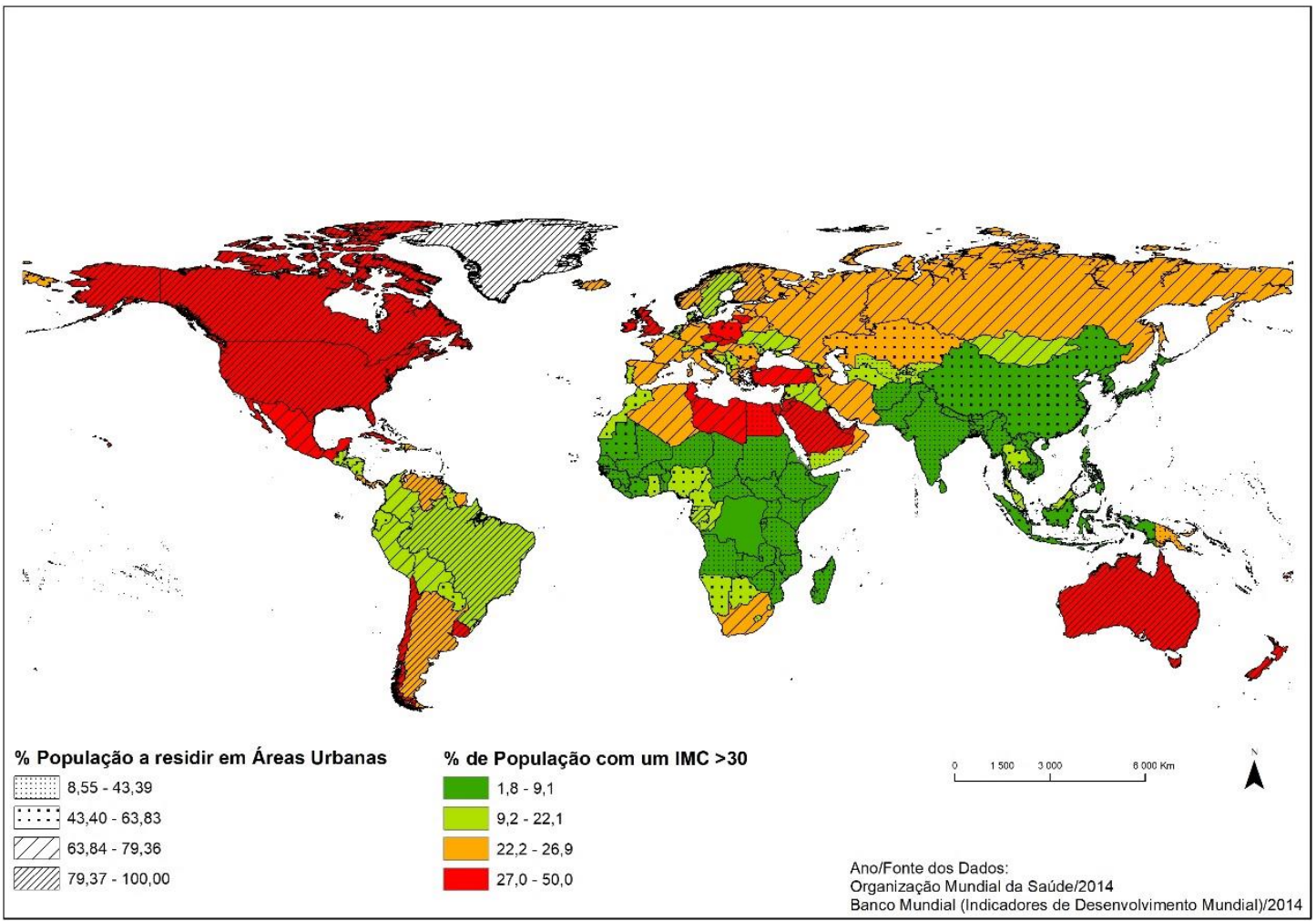

Fig. 2 - Obesidade e Urbanização a nível Mundial

As taxas de obesidade são tão elevadas, quer em países com elevada renda económica ou baixa, que esta se tornou um dos mais importantes tópicos de saúde pública a nível mundial (Fig. 2).

\section{Portugal}

Nas sete regiões ${ }^{1}$ de Portugal (NUT $\|^{2}$ ), a media de densidade de população urbana era $622.53 \mathrm{hab} / \mathrm{km}^{2}$ em 2011 e a percentagem média de população com um IMC acima de 30 era 14.89 in 2005/6. Neste período, Lisboa e Vale do Tejo era a região que apresentava a percentagem mais elevada de população com um IMC acima de 30 e a mais elevada percentagem de população a viver em áreas urbanas.

Em 2014 a percentagem de população com um IMC acima de 30 aumentou para $17,34 \%$ mas a densidade de população urbana diminuiu para $613,76 \mathrm{hab} / \mathrm{km}^{2}$. Enquanto em Lisboa e Vale do Tejo a percentagem de indivíduos obesos diminuiu,

\footnotetext{
${ }^{1}$ Norte, Centro, Lisboa e Vale do Tejo, Alentejo, Algarve, Região Autónoma dos Açores (RAA) e Região Autónoma da Madeira (RAM).

${ }^{2}$ A classificação NUTS (Nomenclatura Unitária Territorial para Estatística) é o Sistema hierárquico para a divisão económica do território na União Europeia com o propósito de: recolher, desenvolver e harmonizar as estatísticas regionais europeias e as analises socioeconómicas das regiões. A NUTS 1: regiões socioecónicas major, NUTS 2: regiões base para a aplicação de políticas regionais, NUTS 3: pequenas regiões para diagnósticos específicos. 21. Eurostat. NUTS - Nomenclature of Territorial Units for Statistics. 2015 07/01/2017]; Available from: http://ec.europa.eu/eurostat/web/nuts/overview.
} 
nas regiões do Centro, Alentejo, Região Autónoma do Açores e Região Autónoma da Madeira aumentou.
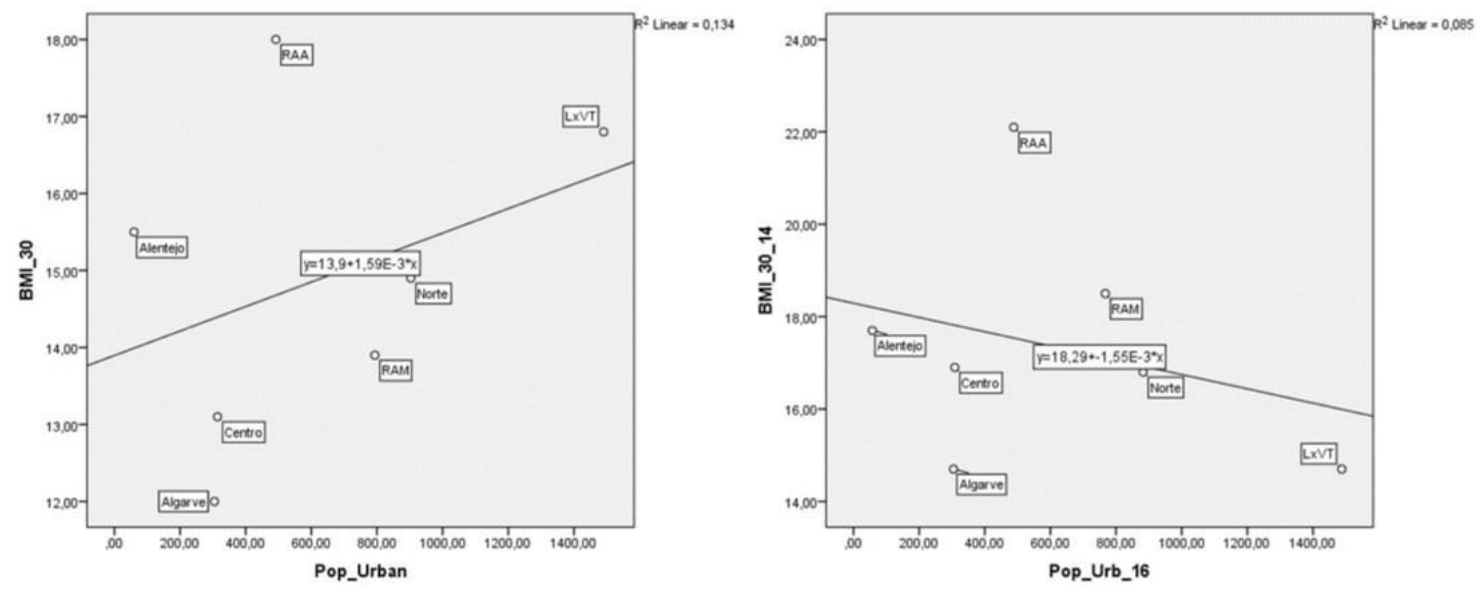

Fig. 3 - Scatter Plot com a Linha de Regressão e respetivo Coeficiente de Determinação (\% População com um IMC acima de $30 \%$ de População a viver em Áreas Urbanas - Regiões Portugal)

[Nota: O scatter à esquerda diz respeito a informação sobre o IMC de 2005-6 e densidade de população urbana de 2011; O scatter à direita refere-se a informação de IMC de 2014 e densidade de população urbana de 2016]

Em Portugal, os resultados com dados de 2005-6 mostram uma correlação entre a percentagem de população com um IMC acima de 30 e a densidade de população urbana $(r=0.366)$. De acordo com o coeficiente de determinação $\left(R^{2}=0.134\right)$, mais de $13 \%$ da proporção da variância na variável dependente (IMC) era previsível pela variável independente (população urbana). A Região Autónoma dos Açores, considerada como uma Outermost Region ${ }^{3}$ pela União Europeia, isto é, uma das regiões socioeconomicamente mais desfavorecidas na União Europeia [22], apresentam a mais elevada prevalência de obesidade (Fig.3). Comida altamente calórica é mais barata por isso, mais acessível para os indivíduos de níveis socioeconómicos mais baixos, o que poderá explicar esta alta prevalência de obesidade nos Açores [23].

No entanto, os dados de 2014 mostram uma correlação negativa entre a percentagem de população com IMC acima de 30 e a densidade de população urbana o que indica que a direção da relação entre ambas as variáveis mudou $(r=-0.29)$.

A Fig. 4 representa a densidade da população urbana e a percentagem de população com um IMC acima de 30, em Portugal nos momentos estudados. A análise do mapa

\footnotetext{
3 “...the outermost regions (ORs), have to deal with a number of difficulties related to their geographical characteristics, in particular: remoteness, insularity, small size, difficult topography and climate. They are economically dependent on a few products (often agricultural products or natural resources). These features act as constraints on their future development potential." 22. $\quad$ EU, Outermost Regions. 2017

http://www.europarl.europa.eu/atyourservice/en/displayFtu.html?ftuld=FTU_5.1.7.html.
}

REVISTA GEONORTE, V.9, N.32, p.06-23, 2018.

DOI: 10.21170/geonorte.2018.V.9.N.32.06.23

(ISSN 2237 - 1419) 


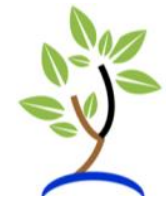

referente aos dados mais antigos, possibilita a identificação de que a região com a mais elevada densidade de população urbana era Lisboa e Vale do Tejo, que também apresentava a mais alta prevalência de população obesa, como verificado na análise estatística feita antes. Comparando este mapa com o mapa produzido com os dados mais recentes, pode-se afirmar que Lisboa e Vale do Tejo foi a única região que melhorou ao reduzir a sua percentagem de indivíduos obesos apesar de ser a região com a mais elevada percentagem de população urbana.
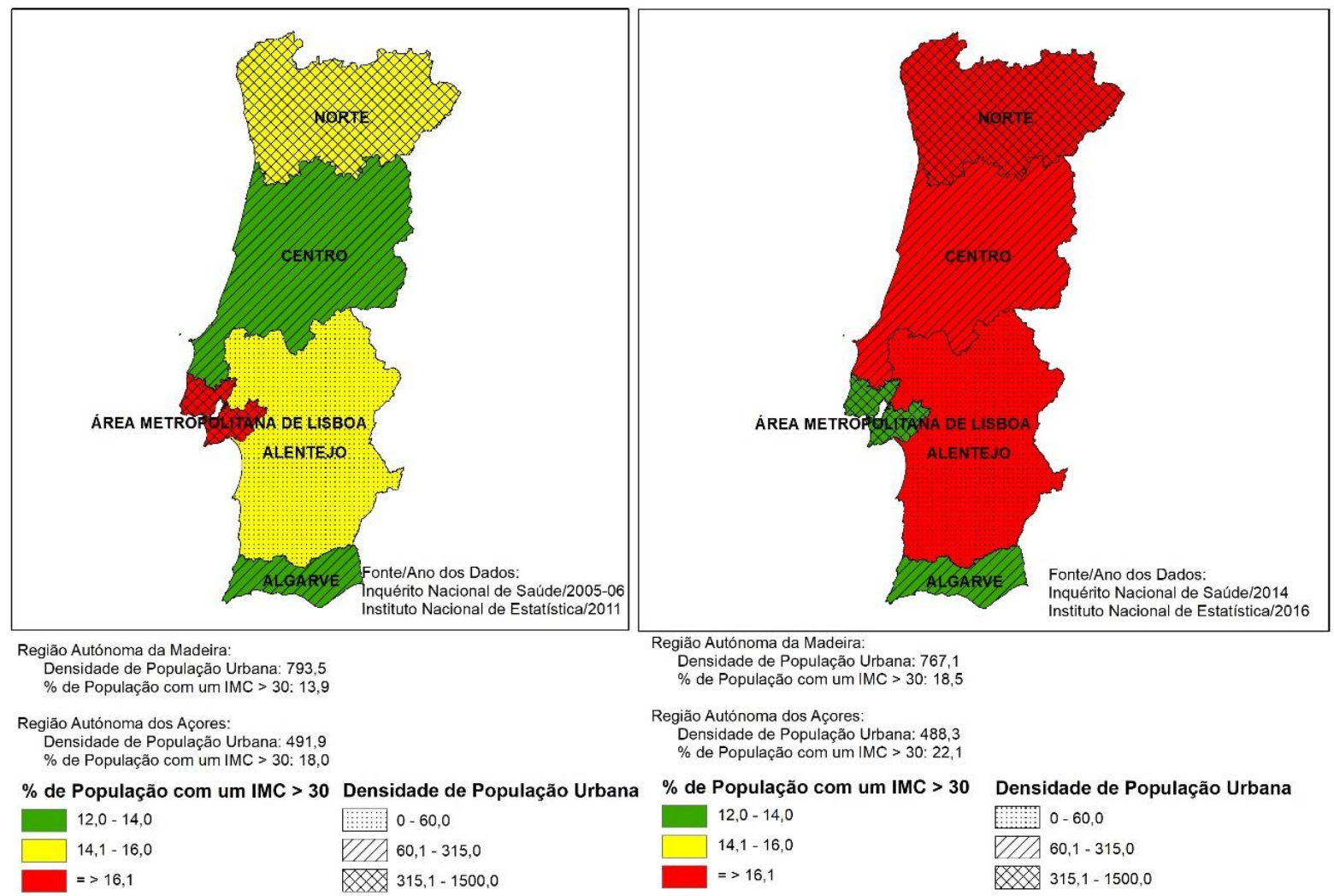

Fig. 4 - Obesidade e Urbanização em Portugal

\section{DISCUSSÃO}

\section{Mundo}

Os resultados de vários estudos mostram a relação entre o ambiente urbano e o excesso de peso e obesidade. Por exemplo, Gordon-Larsen, Wang [24] analisaram as tendências de IMC e Circunferência da Cintura (CC) na China utilizando dados dos últimos 20 anos. Os seus resultados indicam que uma maior incidência de excesso de peso estava associada a um aumento nos scores de urbanização [24]. No estudo realizado por Zhai, Du [25], os resultados mostram que áreas mais urbanizadas estavam associadas com mais elevados consumos de óleos bastante calóricos como fonte de energia principal, maiores consumos de alimentos de origem animal e menos refeições preparadas em casa, enquanto a fonte de energia principal nas áreas de 


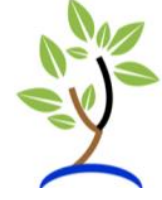

menor urbanização eram os cereais e consumindo-se aí maioritariamente refeições preparadas em casa.

Um estudo realizado com uma amostra de adultos europeus e africanos subsarianos mostra que a urbanização estava significativamente associada com CC e IMC mais elevados dentre os camaronenses urbanos e homens franceses [26]. Outro estudo realizado na capital do Burkina Faso mostra que os residentes em áreas densas em edifícios altos tinham maior probabilidade de serem obesos quando comparados com residentes de áreas com baixa densidade de edifícios não estruturados [20]. Os resultados do estudo "The Peru Migran" também concluiu que não só a população urbana, mas também os migrantes do meio rural para o urbano apresentavam maior incidência de obesidade quando comparado com a população rural [27].

De acordo com uma revisão sistemática e meta-análise, no sudoeste asiático a associação ente viver em áreas urbanas e obesidade era mais forte em países com um Rendimento Nacional Bruto per capita baixo [16], onde as diferenças ente áreas urbanas e rurais são mais evidentes [28]. Isto levou os autores a concluir que os impactos negativos da urbanização na saúde são particularmente mais pronunciados nos países em desenvolvimento [16]. O estudo de Neuman, Kawachi [29] sobre a relação entre a residência urbana e IMC nos países de baixa renda económica mostra que todos os países incluídos na análise apresentam elevadas prevalências de obesidade nas áreas urbanas e uma associação estatisticamente significativa entre viver em áreas urbanas e elevados IMC.

Nos países mais desenvolvidos, a obesidade e a urbanização relacionam-se de formas diferentes. Em 2007, um estudo realizado em 10 países europeus avaliou a prevalência de obesidade de acordo com o tipo de residência da população (urbana ou rural) e não encontrando diferenças relevantes. Os investigadores discutem que nestes países, os residentes em áreas urbanas ou rurais têm um acesso semelhante à comida e ao transporte. Adicionalmente, os autores sugerem que os conceitos de rural e urbano nos países europeus poderá não se assemelhar aos do países de baixa renda económica onde existem maiores diferenças entre as áreas urbanas e rurais [28]. Outro fator que poderá ter tido um papel importante nestes resultados foi o Produto Interno Bruto (PIB). Os países em desenvolvimento com um elevado PIB apresentam prevalências de obesidade mais baixas, enquanto na Europa acontece o oposto - países com menor PIB têm prevalências de obesidade mais elevadas uma vez que em países com elevado PIB é mais fácil o acesso a comida saudável e a locais para a prática de atividade física [28].

Num relatório recente sobre políticas de obesidade nos EUA, uma das principais preocupações são os designados "desertos alimentares", ou seja, áreas onde não existem supermercados disponíveis a menos de uma milha (aproximadamente $1,6 \mathrm{~km})$, em áreas urbanas e até 10 milhas em áreas rurais. Acredita-se que mais de 29 milhões de americanos vivam em "desertos alimentares" lidando com a falta de acesos a comida saudável [30].

\section{Portugal}


De acordo com um estudo publicado no Jornal da Direção Geral da Saúde Portuguesa, baseado em dados de $2009,27,8 \%$ das mulheres e $53,3 \%$ dos homens eram pré-obesos. Além disso, 10,4\% das mulheres e 11,2\% dos homens eram obesos [31]. Em 2013, em Portugal, 28,7\% dos rapazes e 10,6\% das raparigas com menos de 20 anos, $20,9 \%$ dos homens e $23,4 \%$ das mulheres com 20 ou mais anos eram obesas [1]. Estes números são muito preocupantes e colocam Portugal como um dos países com a maior proporção de crianças obesas [32].

Em Portugal, existem poucos estudos sobre obesidade e urbanização. Em 2009, um estudo liderado por Santana P. com o objetivo "(...) the contribution of the local environment and personal attributes to the risk of weight gain in LMA ${ }^{4}$ neighbourhoods (...)" teve como resultado principal que as pessoas que viviam no tercil com maior densidade populacional aumentavam a sua chance de ficar obesas [19].

Num estudo mais recente focado na associação entre as perceções dos pais da sua própria área de residência e o peso das crianças, realizado no Porto - a segunda maior área urbana em Portugal. Os autores concluíram que as opiniões dos pais sobre os aspetos sociais e biofísicos da sua vizinhança tinham impacto no peso das crianças. Por exemplo, a crença dos pais que não havia passeios de qualidade na vizinhança estava associada significativamente à obesidade infantil [17]. No mesmo ano, Nogueira, Ferrao [33] concluíram que a obesidade não só é um problema de saúde como também um tópico de planeamento urbano uma vez que, como mostram os resultados deste estudo, as perceções negativas dos pais sobre o ambiente da sua vizinhança aumentam a chance das crianças se tornarem obesas, principalmente as raparigas.

\section{Conclusão}

A maior conclusão deste estudo é que a urbanização, mais especificamente os estilos de vida urbanos, podem levar a alterações na composição corporal dos indivíduos. Países de elevada renda económica têm uma percentagem maior de população urbano e também das maiores percentagens de população com um IMC acima de 30 enquanto países com baixa renda económica são menos urbanizados e apresentam mais baixas percentagens de indivíduos obesos.

No entanto, a relação entre urbanização e obesidade difere entre países de acordo com o enquadramento de cada país relativamente ao nível do desenvolvimento social e económico. Apesar de países com menores produtos internos brutos apresentarem menos prevalência de obesidade no geral, a associação entre a urbanização e a obesidade é mais evidente em países com rendas económicas mais baixas onde o produto interno bruto per capita é menor. Nos países de elevada renda económica, como os EUA, as especificidades de uso do solo poderão determinar as escolhas individuais de consumo alimentar. Por exemplo, grandes distâncias entre as residências e as lojas de produtos alimentares poderão ser consideradas como barreiras para ter hábitos alimentares mais saudáveis.

${ }^{4}$ LMA: Lisbon Metropolitan Area

REVISTA GEONORTE, V.9, N.32, p.06-23, 2018.

DOI: 10.21170/geonorte.2018.V.9.N.32.06.23

(ISSN 2237 - 1419) 


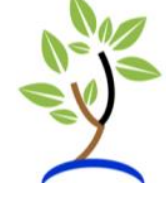

Os resultados sugerem que em Portugal, até 2006, as regiões com densidades mais elevadas de população urbana apresentavam maiores prevalências de obesidade, mas esta tendência mudou, isto é, dados mais recentes mostram que a percentagem mais elevada de indivíduos obesos vive em regiões com menores densidade de população urbana. Até ao momento, isto ainda não foi discutido na literatura disponível que se tem direcionado para a associação entre as perceções do ambiente urbano e a composição corporal das crianças.

É necessária mais investigação para aprofundar a compreensão das principais determinantes da obesidade a nível mundial e em Portugal porque o conhecimento sobre os principais fatores de risco para a obesidade, a diferentes níveis de análise, é por vezes contraditório e continua por clarificar.

Este estudo foi desenvolvido usando dados gratuitamente disponíveis que, apesar de recolhidos em fontes fidedignas, apresentam lacunas o que pode ser considerado como um viés na análise realizada. No que diz respeito ao território português e nas dimensões demográficas, deveriam ter sido utilizados dados mais desagregados quando se analisa a relação entre urbanização e obesidade; no entanto, a informação sobre o IMC dos indivíduos apenas se encontra disponível ao nível da NUTII.

Finalmente, o planeamento urbano deveria ter em consideração as questões de saúde e, talvez mais importante ainda, os peritos em saúde pública não deveriam desconsiderar a importância dos aspetos ambientais como as condições de trabalho, transporte e de vida no geral, quando desenvolvem e implementam as intervenções direcionadas à redução da prevalência da obesidade.

\section{Financiamento:}

Este trabalho foi financiado pela Fundação para a Ciência e Tecnologia (bolsa de doutoramento SFRH/BD/133140/2017).

\section{REFERENCIAS}

1. $\mathrm{Ng}, \mathrm{M}$., et al., Global, regional, and national prevalence of overweight and obesity in children and adults during 1980-2013: a systematic analysis for the Global Burden of Disease Study 2013. The Lancet, 2014. 384(9945): p. 766-781

2. Caballero, B., The global epidemic of obesity: an overview. Epidemiol Rev, 2007. 29: p. 1-5 https://www.ncbi.nlm.nih.gov/pubmed/17569676; http://epirev.oxfordjournals.org/content/29/1/1.full.pdf.

3. Patil, R.R., Urbanization as a determinant of health: a socioepidemiological perspective. Soc Work Public Health, 2014. 29(4): p. 335-41 https://www.ncbi.nlm.nih.gov/pubmed/24871771.

4. Sui, D.Z., Musings on the Fat City: Are Obesity and Urban Forms Linked? Urban Geography, 2013. 24(1): p. 75-84 
5. Malik, V.S., W.C. Willett, and F.B. Hu, Global obesity: trends, risk factors and policy implications. Nat Rev Endocrinol, 2013. 9(1): p. 13-27 https://www.ncbi.nlm.nih.gov/pubmed/23165161.

6. Huang, T.T., et al., A systems-oriented multilevel framework for addressing obesity in the 21st century. Prev Chronic Dis, 2009. 6(3): p. A82 http://www.ncbi.nlm.nih.gov/pubmed/19527584;

https://www.ncbi.nlm.nih.gov/pmc/articles/PMC2722412/pdf/PCD63A82.pdf.

7. Cassel, K.D., Using the Social-Ecological Model as a research and intervention framework to understand and mitigate obesogenic factors in Samoan populations. Ethn Health, 2010. 15(4): p. 397-416 https://www.ncbi.nlm.nih.gov/pubmed/20560084.

8. James, W.P., The fundamental drivers of the obesity epidemic. Obes Rev, 2008. 9 Suppl 1: p. 6-13 https://www.ncbi.nlm.nih.gov/pubmed/18307693; http://onlinelibrary.wiley.com/doi/10.1111/j.1467-789X.2007.00432.x/abstract.

9. Mendes, L.L., et al., Individual and environmental factors associated for overweight in urban population of Brazil. BMC Public Health, 2013. 13: p. 988 https://www.ncbi.nlm.nih.gov/pubmed/24143958;

https://www.ncbi.nlm.nih.gov/pmc/articles/PMC3854448/pdf/1471-2458-13-988.pdf.

10. Popkin, B.M. and C.M. Doak, The Obesity Epidemic Is a Worldwide Phenomenon. Nutrition Reviews, 1998. 56(4): p. 106-114 http://nutritionreviews.oxfordjournals.org/content/56/4/106.

11. World Bank Group. United Nations Population Division. World Urbanization Prospects: 2014 Revision. 2018 12/06/2018]; Available from: https://data.worldbank.org/indicator/SP.URB.TOTL.IN.ZS.

12. WHO, Urbanization and health. Bulletin of the World Health Organization (BLT), 2010. 88(4): p. 241-320 http://www.who.int/bulletin/volumes/88/4/10010410/en/.

13. Kumanyika, S., et al., Obesity prevention: the case for action. Int J Obes Relat Metab Disord, 2002. 26(3): p. 425-36 http://www.ncbi.nlm.nih.gov/pubmed/11896500; http://www.nature.com/ijo/journal/v26/n3/pdf/0801938a.pdf.

14. Popkin, B.M., The nutrition transition in low-income countries: an emerging $\begin{array}{lllll}\text { crisis. } \quad \text { Nutr } & \text { Rev, } & \text { 52(9): } & \text { p. }\end{array}$ https://www.ncbi.nlm.nih.gov/pubmed/7984344.

15. Popkin, B. and P. Gordon-Larsen, The nutrition transition: worldwide obesity dynamics and their determinants. International Journal of Obesity, 2004. 28: p. S2-S9 
A RELAÇÃO ENTRE A URBANIZAÇÃO E A EPIDEMIA DA OBESIDADE A NÍVEL MUNDIAL E EM PORTUGAL

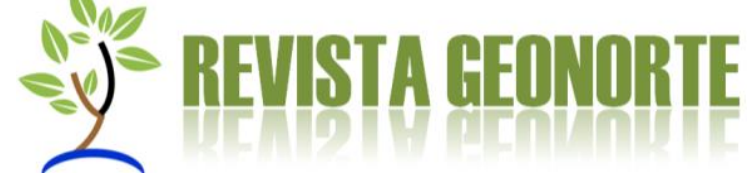

16. Angkurawaranon, C., et al., Urban environments and obesity in southeast Asia: a systematic review, meta-analysis and meta-regression. PLoS One, 2014. 9(11): p. e113547 https://www.ncbi.nlm.nih.gov/pubmed/25426942

https://www.ncbi.nlm.nih.gov/pmc/articles/PMC4245122/pdf/pone.0113547.pdf.

17. Ferrao, M.M., et al., Association between parental perceptions of residential neighbourhood environments and childhood obesity in Porto, Portugal. Eur J Public Health, 2013. 23(6): p. 1027-31 https://www.ncbi.nlm.nih.gov/pubmed/23325125; http://eurpub.oxfordjournals.org/content/eurpub/23/6/1027.full.pdf.

18. Goryakin, Y. and M. Suhrcke, Economic development, urbanization, technological change and overweight: what do we learn from 244 Demographic and Health Surveys? Econ Hum Biol, 2014. 14: p. 109-27 https://www.ncbi.nlm.nih.gov/pubmed/24457038; cdn.com/S1570677X13001275/1-s2.0-S1570677X13001275main.pdf? tid=97d16852-c06a-11e6-882600000aacb35e\&acdnat $=1481547652$ ea3955f294d50e534568cd6dd8fca6d2.

19. Santana, P., R. Santos, and H. Nogueira, The link between local environment and obesity: a multilevel analysis in the Lisbon Metropolitan Area, Portugal. Soc Sci Med, 2009. 68(4): p. 601-9 https://www.ncbi.nlm.nih.gov/pubmed/19135287

http://www.sciencedirect.com/science/article/pii/S0277953608006084.

20. Ouedraogo, H.Z., et al., Socio-spatial disparities of obesity among adults in the urban setting of Ouagadougou, Burkina Faso. Public Health Nutr, 2008. 11(12): p. 1280-7 https://www.ncbi.nlm.nih.gov/pubmed/18503721; https://www.cambridge.org/core/services/aop-cambridgecore/content/view/61C4B78C9205C75E0EFE3EF51E78A904/S1368980008002504a .pdf/div-class-title-socio-spatial-disparities-of-obesity-among-adults-in-the-urbansetting-of-ouagadougou-burkina-faso-div.pdf.

21. Eurostat. NUTS - Nomenclature of Territorial Units for Statistics. 2015 07/01/2017]; Available from: http://ec.europa.eu/eurostat/web/nuts/overview.

22. EU, Outermost Regions. 2017 http://www.europarl.europa.eu/atyourservice/en/displayFtu.html?ftuld=FTU 5.1.7.htm I.

23. de Ávila, V.M.L., Prevalência de Excesso de Peso e de Obesidade em Crianças entre os 6 e os 10 anos de ldade no Concelho de Angra do Heroísmo, in Faculdade Ciências da Saúde. 2008, Universidade da Beira Interior. p. 103 http://ubibliorum.ubi.pt/bitstream/10400.6/727/1/Prevalencia\%20de\%20Excesso\%20 de\%200besidade\%20Infantil.pdf. 
A RELAÇÃO ENTRE A URBANIZAÇÃO E A EPIDEMIA DA OBESIDADE A NÍVEL MUNDIAL E EM PORTUGAL

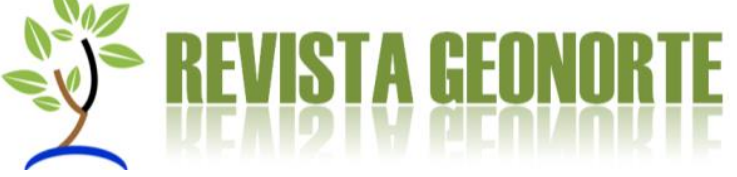

24. Gordon-Larsen, P., H. Wang, and B.M. Popkin, Overweight dynamics in Chinese children and adults. Obes Rev, 2014. 15 Suppl 1: p. 37-48 https://www.ncbi.nlm.nih.gov/pubmed/24341757;

http://onlinelibrary. wiley.com/store/10.1111/obr.12121/asset/obr12121.pdf?v=1\&t=iw m33vv7\&s=22969c788eddb0c7d54996aa30b4b620aebbff2e.

25. Zhai, F.Y., et al., Dynamics of the Chinese diet and the role of urbanicity, 1991 2011. Obes Rev, 2014. 15 Suppl 1: p. $16-26$ https://www.ncbi.nlm.nih.gov/pubmed/24341755;

http://onlinelibrary.wiley.com/store/10.1111/obr.12124/asset/obr12124.pdf?v=1\&t=iw m357y7\&s=1ff9333d00e30b14691c3c53b5626a52a947deb8.

26. Fezeu, L., et al., Waist circumference and obesity-related abnormalities in French and Cameroonian adults: the role of urbanization and ethnicity. Int $\mathrm{J}$ Obes (Lond), 2010. 34(3): p. 446-53 https://www.ncbi.nlm.nih.gov/pubmed/20065972; http://www.nature.com/ijo/journal/v34/n3/pdf/ijo2009256a.pdf.

27. Carrillo-Larco, R.M., et al., Obesity risk in rural, urban and rural-to-urban migrants: prospective results of the PERU MIGRANT study. Int J Obes (Lond), 2016. 40(1): $\quad$ p. $181-5 \quad$ https://www.ncbi.nlm.nih.gov/pubmed/26228458; https://www.ncbi.nlm.nih.gov/pmc/articles/PMC4677453/pdf/ijo2015140a.pdf.

28. Peytremann-Bridevaux, I., D. Faeh, and B. Santos-Eggimann, Prevalence of overweight and obesity in rural and urban settings of 10 European countries. Prev Med, 2007. 44(5): p. 442-6 https://www.ncbi.nlm.nih.gov/pubmed/17258803.

29. Neuman, M., et al., Urban-rural differences in BMI in low- and middle-income countries: the role of socioeconomic status. Am J Clin Nutr, 2013. 97(2): p. 428-36 https://www.ncbi.nlm.nih.gov/pubmed/23283503;

https://www.ncbi.nlm.nih.gov/pmc/articles/PMC3742298/pdf/ajcn97428.pdf.

30. Segal, L.M., J. Rayburn, and A. Martín, The State of Obesity, in Obesity Policies. 2016, Robert Wood Jonhson Foundation http://stateofobesity.org/files/stateofobesity2016.pdf.

31. Graça, P. and M.J. Gregório, Estratégia para a promoção da alimentação saudável em Portugal. Portugal Saúde em Números, 2015. 4: p. 37-41

32. Nogueira, $\mathrm{H}$., et al., The associations of SES, obesity, sport activity, and perceived neighborhood environments: is there a model of environmental injustice penalizing Portuguese children? Am J Hum Biol, 2013. 25(3): p. 434-6 https://www.ncbi.nlm.nih.gov/pubmed/23559457;

http://onlinelibrary.wiley.com/doi/10.1002/ajhb.22384/abstract. 
A RELAÇÃO ENTRE A URBANIZAÇÃO E A EPIDEMIA

DA OBESIDADE A NÍVEL MUNDIAL E EM PORTUGAL

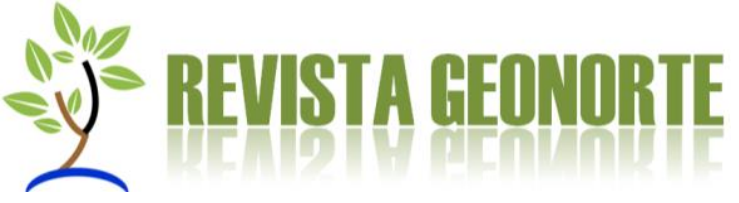

33. Nogueira, H., et al., Perceptions of neighborhood environments and childhood obesity: evidence of harmful gender inequities among Portuguese children. Health Place, 2013. 19: p. 69-73 https://www.ncbi.nlm.nih.gov/pubmed/23201911; http://www.sciencedirect.com/science/article/pii/S1353829212001840.

Submetido em: 22/05/2018

Aceito para publicação em: 11/06/2018 